\title{
Establishment of Multi-medium PDEs Model and Temperature Distribution
}

\author{
Yifei Ma ${ }^{1}$, Ziqing Hao ${ }^{1}$, Qiyan Niu ${ }^{1}$ \\ ${ }^{1}$ Statistic Institute, Shanxi University of Finance and Economics, wucheng road, Taiyuan, China
}

Keywords: Thermal partial differential equation; Multi-medium PDE model; Temperature distribution; Finite difference method

Abstract: In this paper, a multi-medium partial differential equation (PDE) model is established according to the temperature change data of the outer skin of the dummy in the thermal effect simulation experiment, considering the influence of density $(\rho)$, specific heat capacity $(\mathrm{C})$, thermal conductivity $(\lambda)$, thickness $(\mathrm{h})$ of different materials. In this paper, it is found that under certain conditions, the combination of Fourier law and the partial differential equation has a good effect on the heat conduction effect and temperature distribution in unsteady time. At the same time, while achieving the goal of thermal protection, it can reduce the cost and reduce the $\mathrm{R} \& \mathrm{D}$ cycle.

\section{Introduction}

With the increasing demand of high temperature work, the demand for thermal protection special clothes is higher and higher. In order to optimize the clothing material structure, the temperature distribution in the outer skin of the dummy is obtained by simulating the dummy with a constant temperature of $37 \mathrm{C}$.

To simplify the problem, the thermal protective clothing materials are divided into I, II, III and IV layers. The material parameters are as follows:

Table 1 material parameter values

\begin{tabular}{cccc}
\hline stratification & $\begin{array}{c}\rho \\
\left(\mathrm{kg} / \mathrm{m}^{3}\right)\end{array}$ & $\begin{array}{c}\mathrm{C} \\
\left(\mathrm{J} /\left(\mathrm{kg} \cdot{ }^{\circ} \mathrm{C}\right)\right)\end{array}$ & $\begin{array}{c}\lambda \\
\left(\mathrm{W} /\left(\mathrm{m} \cdot{ }^{\circ} \mathrm{C}\right)\right.\end{array}$ \\
\hline I & 300 & 1377 & 0.082 \\
II & 862 & 2100 & 0.37 \\
III & 74.2 & 1726 & 0.045 \\
IV & 1.18 & 1005 & 0.028 \\
\hline
\end{tabular}

By using the thermal equation model, the temperature distribution model of the dummy is obtained. 


\section{Establishment of multi-medium partial differential equation (PDE) model}

\subsection{The basic theory of Fourier}

In the heat conduction process, the heat through a given cross-section is proportional to the temperature change rate and cross-section area perpendicular to the cross-section, and the direction of heat transfer is opposite to the direction of the temperature rise. Fourier of heat conduction is:

$$
\frac{Q}{S}=\frac{\partial T}{\partial x}
$$

The general mathematical formula is:

$$
Q=-\lambda S \frac{\partial T}{\partial x}
$$

Among them, $\frac{d Q}{d t}$ (one point on $\mathrm{Q}$ ) is the heat conduction rate, the unit is $\mathrm{w}$. $\mathrm{S}$ is the heat transfer area, the unit is $m^{2}$. The temperature of T is $\mathrm{K}$. X is the coordinate on the heat conduction surface and the unit is $\mathrm{m}$.

\subsection{Analysis of actual situation based on Fourier}

Each layer of the media is different, resulting in different heat conductivity, considering the thickness of the media and other factors, resulting in separate heat transfer, using the flat-wall multi-layer heat conduction model to study. We set the thermal conductivity $\lambda_{1}, \lambda_{2}, \lambda_{3}, \lambda_{4}$ of layers I, II, III, IV to be affected by the parameters of the material medium. The greater the thermal conductivity, the better the thermal conductivity of the object. For solid media, there is a linear change in temperature. When the pressure is moderate, the gaseous medium increases with the increase of temperature.

In the flat-wall multilayer heat conduction model, the area of each layer is $\mathrm{S}$, and the thickness of each layer is $L_{i}(\mathrm{i}=1,2,3,4)$, as showned in Figure 1.

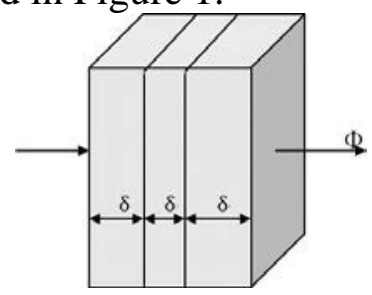

Fig. 1 multilayer heat conduction model with flat wall

The Fourier model at each level is:

$$
\begin{gathered}
\text { First floor, } \frac{Q}{S}=\frac{\lambda_{1}}{L_{1}} \Delta t_{1}, \Delta t_{1}=t_{2}-t_{1}=\frac{Q L_{1}}{S \lambda_{1}} \text { (1), } \\
\text { Second floor, } \frac{Q}{S}=\frac{\lambda_{2}}{L_{2}} \Delta t_{2}, \Delta t_{2}=t_{3}-t_{2}=\frac{Q L_{2}}{S \lambda_{2}}(2), \\
\text { Third floor, } \frac{Q}{S}=\frac{\lambda_{3}}{L_{3}} \Delta t_{3}, \Delta t_{3}=t_{4}-t_{3}=\frac{Q L_{3}}{S \lambda_{3}}(3), \\
\text { Fourth floor, } \frac{Q}{S}=\frac{\lambda_{4}}{L_{4}} \Delta t_{4}, \Delta t_{4}=t_{5}-t_{4}=\frac{Q L_{4}}{S \lambda_{4}}(4),
\end{gathered}
$$


$\mathrm{Q}$ is the heat conduction rate, which is undetermined in the flat-wall multi-layer heat conduction model. Therefore, the following equations (1), (2), (3) (4) can be obtained:

$$
Q=\frac{S \Delta \mathrm{t}}{\frac{\mathrm{L}_{1}}{\lambda_{1}}+\frac{L_{2}}{\lambda_{2}}+\frac{L_{3}}{\lambda_{3}}+\frac{L_{4}}{\lambda_{4}}}=\frac{\Delta \mathrm{t}}{\sum \frac{\mathrm{L}}{S \lambda}}
$$

\subsection{Theoretical basis of heat conduction equation}

In this paper, the heat conduction equation is used for modeling and analysis. The heat conduction in three-dimensional uniform media can be expressed by the following equation:

$$
\frac{\partial u}{\partial t}+\operatorname{div}(U u)=k\left(\frac{\partial^{2} u}{\partial x^{2}}+\frac{\partial^{2} u}{\partial y^{2}}+\frac{\partial^{2} u}{\partial z^{2}}\right)=k\left(u_{x x}+u_{y y}+u_{z z}\right)
$$

Among them: $u=u(x, y, z, t)$ is temperature and the function of time variable $\mathrm{t}$ and changing space position; $\frac{\partial u}{\partial t}$ is the rate of change of temperature to time at a point in space; $u_{x x}, u_{y y}$ and $u_{z z}$ is the second derivative of temperature to three space coordinate axes; $\mathrm{k}$ is the thermal diffusivity, depending on the material's thermal conductivity, density and heat capacity.

Consider one-dimensional heat conduction equation such as formula (1).

$$
\frac{\partial u}{\partial t}+\operatorname{div}(U u)=k\left(\frac{\partial^{2} u}{\partial L^{2}}\right)=k(u)
$$

$\mathrm{K}$ is the thermal diffusivity, and its value will be affected by the thermal conductivity, density and specific heat capacity of the material. In other words, the independent variable in the model is the thickness of each layer $\mathrm{L}$, and the dependent variable is the thermal conductivity rate, that is, the variable with parameter time.

\subsection{Solution of K value}

Because of the changed composition of the medium, the specific heat and heat conductivity will affect the heat transfer rate, and the density of the material will have a greater impact on the solid-state heat transfer. The magnitude of the effect is expressed by $\mathrm{K}$.

The relationship between $K$ and thickness $L$ is not simply linear, because with the increase in temperature, the thermal movement of the molecule is accelerated. At the same time, the heat conduction of the air in the pore and the radiation between the pore walls are also increased, resulting in the change of $\mathrm{K}$, and $\mathrm{K}$ is also related to the material itself. The thermal diffusivity is the ratio of thermal conductivity $\lambda$ to the product of specific heat capacity $\mathrm{c}$ and density $\rho$.

$$
K=\frac{\lambda}{\mathrm{c} \rho}
$$

The K values of various materials are shown below,

Table $2 \mathrm{~K}$ values of various materials

\begin{tabular}{cc}
\hline stratification & $\mathrm{K}$ \\
\hline I & $1.98499 \mathrm{E}-07$ \\
II & $2.04397 \mathrm{E}-07$ \\
III & $3.51373 \mathrm{E}-07$ \\
IV & $2.36108 \mathrm{E}-05$ \\
\hline
\end{tabular}




\section{Analysis based on steady and unsteady differential equations}

\subsection{Basic data processing}

The dummy lateral temperature change data is drawn by MATLAB, as shown in Figure 2.

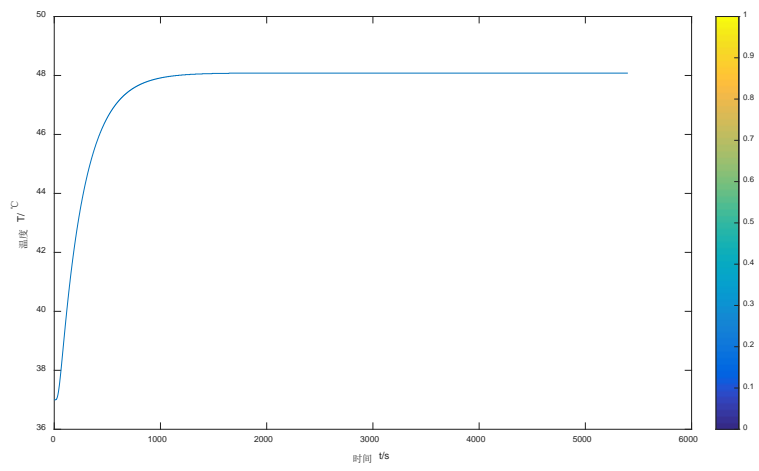

Fig. 2 Changes of temperature on the outer side of the dummy skin

\subsection{The conditions for determining the differential equations of heat}

Only partial differential equations are not sufficient to determine the law of temperature variation with space, because temperature is also related to its initial state and the constraints it is subject to at the boundary under certain conditions. In the unsteady state problem, the definite solution conditions include the initial conditions and boundary conditions, assuming that the external temperature is constant at 75 degrees Celsius, and the dummy is adiabatic, so the boundary conditions are adiabatic and constant heat flow.

One dimensional heat conduction equation can be written as:

$$
\mathrm{Ut}(\mathrm{x}, \mathrm{t})=K^{2 *} \mathrm{Uxx}(\mathrm{x}, \mathrm{t}) \quad \mathrm{a}<x<\mathrm{b} \quad \text { ts }<t<\mathrm{tf}
$$

Among them, $\mathrm{K}$ refers to the thermal diffusivity, $\mathrm{f}$ is the initial condition and $\mathrm{U}$ is the solution of the equation. The initial condition is the temperature distribution of the material at the initial time.

Implicit function:

$$
T(x, 0)=\psi(x)
$$

$\psi(x)$ A known function in a formula.

The initial temperature is set at $75 \mathrm{C}$ by studying the different media sections of I, II, III and IV layer, ignoring the influence of various factors such as thermal radiation and thermal convection between the layers. In order to facilitate calculation, the data is processed by finite difference method, and the continuous data is divided into small sections and then calculated. The problem of continuous temperature distribution, time and material thickness is introduced into a three-dimensional mesh, which is regarded as a set of finite discrete points. The discrete variable function is used to approximately replace the original continuous variable problem. The approximate solution of the discrete point is obtained by solving the equations, and then the approximate solution of the whole continuous region problem is obtained by using the interpolation method to return the original problem from the discrete problem.

Using MATLAB program to solve the thermal differential equation, the $\mathrm{T}$ matrix table with temperature distribution and the three-dimensional diagram of $\mathrm{T}, \mathrm{T}$ and $\mathrm{X}$ are obtained. Essentially, at a time of $t$, the change of $\mathrm{X}$ leads to the difference of $\mathrm{T}$, which is one-dimensional unsteady state. The dimension of temperature distribution is only the thickness of the material $\mathrm{x}$, not the 
two-dimensional distribution and emptiness of the plane points of the material. The three dimensional distribution of each point.

The temperature distribution of the four layer material for high temperature operation is obtained and its segmented function is constructed. The logarithmic processing of time shows that the heat transfer time in the first layer is $0.31 \mathrm{~s}$, in the second layer is $13.81 \mathrm{~s}$, in the third layer is $1.1 \mathrm{~s}$, in the fourth layer is 0.87s. Segment function:

Table 3

\begin{tabular}{c|c|c|c}
\hline & function & \multicolumn{2}{|c}{ parameter } \\
\hline \multirow{2}{*}{ I } & \multirow{2}{*}{$f(x)=-0.1995 \exp (0.623 x)+76.16$} & SSE & 9.725 \\
& & R-square & 0.9835 \\
& \multirow{3}{*}{$f(x)=-0.1384 \exp (0.5235 x)+59.22$} & Adjusted R-square & 0.9815 \\
\hline \multirow{2}{*}{ II } & & SSE & 0.6562 \\
& & Adjusted R-square & 0.9881 \\
& & SSE & 0.9866 \\
\hline \multirow{2}{*}{ III } & $f(x)=1.431 \cos (0.2792 x)+2.424 \sin (0.2792 x)+50.33$ & R-square & 0.9932 \\
& & Adjusted R-square & 0.992 \\
\hline \multirow{3}{*}{ IV } & \multirow{3}{*}{$f(x)=5.9 \cos (0.2994 x)-5.235 \sin (0.2994 x)+44.02$} & SSE & 0.1748 \\
& & R-square & 0.9932 \\
& & Adjusted R-square & 0.992 \\
\hline
\end{tabular}

At the same time, a three-dimensional heat transfer map is obtained. Fig.3 is a three-dimensional heat transfer map of the first layer. The vertical coordinate is time $(\mathrm{t})$, the vertical coordinate is temperature $(\mathrm{T})$, and the abscissa coordinate is thickness (x). In fact, turning the graph into a plane diagram, as shown in Fig. 4, it is obvious that the temperature of the same thickness surface in each layer is the same, that is, $\mathrm{x}$ is only related to the thickness of the medium $\mathrm{L}$, and has nothing to do with the spatial position of the medium. It is a one-dimensional problem.

Table 4 temperature change table

\begin{tabular}{lcccc}
\hline \multicolumn{1}{c}{ stratification } & I & II & III & IV \\
\hline $\begin{array}{l}\text { starting } \\
\text { temperature } /{ }^{\circ} \mathrm{C}\end{array}$ & 75 & 58.2529 & 53.1381 & 51.8867 \\
$\begin{array}{l}\text { outlet } \\
\text { temperature } /{ }^{\circ} \mathrm{C}\end{array}$ & 58.2529 & 53.1381 & 51.8867 & 48.3796 \\
\hline
\end{tabular}

Fig.3Three dimensional map of temperature distribution in layer I 


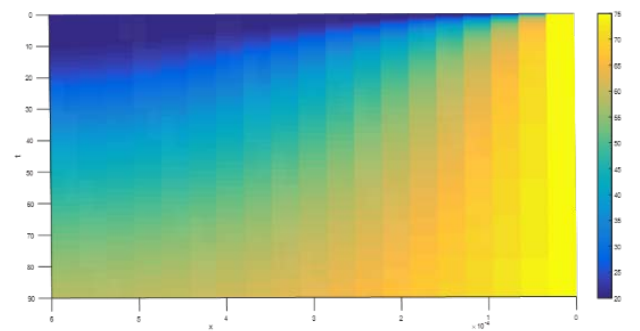

Fig.4 Plane temperature distribution after rotation

\section{References}

[1] Bin Pan. Mathematical modeling of heat transfer in thermal protective clothing and inverse problem of parameter determination [D]. Zhejiang Sci-Tech University, 2017.

[2] Linzhen Lu. Heat transfer model and parameter optimization of multi-layer thermal protective clothing [D]. Zhejiang University of Technology, 2018.

[3] Can Li, Yandong Gao, Suyi Huang. MATLAB numerical calculation of heat conduction problems [J].Journal of Huazhong University of Science and Technology (Natural Science Edition), 2002 (09): 91-93.

[4] Jianliang $\mathrm{Xu}$, Bingshu Tang. Numerical solution of one-dimensional heat conduction equation [J].Journal of Huaiyin Normal University (Natural Science Edition), 2004 (03): 210-214.

[5] Jie Yang. Simulation and Experimental Study of Human Thermal Response to High Temperature Based on Human Body-Clothing-Environment [D]. Tsinghua University, 2016.

[6] Yingchao Li. Computer Experimental Study on Double Medium Heat Transfer in Directional Solidification Process Castings [D]. Shanghai University, 2005.

[7] Zengyuan Guo, Hongye Zhu. Motion and transfer of heat and mass - conservation equation and Fourier law of hot gas [J].Journal of Engineering Thermophysics, 2007 (01): 86-88. 